Case Report

Robin Bhattarai M.S PhD',
Chuan Chen $\mathbf{M D}^{1}$,
Chao Feng Liang MD.PhD',
TengChao Huang $\mathbf{M D}^{1}$,
Hui Wang MD. PhD'
Ying Guo MD. PhD $^{2}$
${ }^{1}$ Department of Neurosurgery, Third Affiliated Hospital of
Sun Yat-Sen University
${ }^{2}$ Head of Department, Department of Neurosurgery, Third
Affiliated Hospital of Sun Yat-Sen University

Address for correspondence:

Prof. Guo Ying MD. PhD

Head of Department

Department of Neurosurgery

Third Affiliated Hospital of Sun Yat-Sen University,

No. 600, Tianhe Road, Guangzhou 510630, Guangdong

Province, PR China

Email:guoyingsysu@hotmail.com

Date submitted : 2/3/2019

Date accepted : 10/5/2019

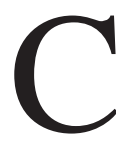
S ICA aneurysms account for 3\%-5\% of all intracranial aneurysms. ${ }^{9}$ These aneurysms bear relatively lower risk due to their location being outside the dura mater and may not result in subarachnoid hemorrhage even when ruptured. Due to the presence of $\mathrm{CS}$, dura mater and other surrounding structures the CS
Nepal Journal of Neurosciences 16:42-47, 2019

\section{Combining Internal Carotid artery Occlusion with Intermediate-Flow Bypass for Treating Large-Giant Cavernous Sinus Segment Aneurysms: Case Report and Review of Literature}

We summarize the treatment effectiveness and experience of a patient who underwent internal carotid balloon occlusion combined with Intermediate-flow bypass as a treatment for large-giant cavernous sinus segment internal carotid artery (CS ICA) aneurysms.

A 62-year-old woman presented with a large aneurysm on the right side of the cavernous sinus with dizziness for about two years and Oculomotor nerve palsy. An extracranialintracranial (EC-IC) Intermediate-flow bypass using a radial artery bypass graft (RABG) and proximal balloon occlusion of the Right ICA were performed. The patient experienced no new neurologic deficit after this treatment. Followup radiologic evaluations using Computed Tomography Angiography revealed complete aneurysm occlusion.

For patients with large-giant CS ICA aneurysms, treatment of ICAocclusion combined with Intermediate-flow superficial temporal artery-Radial artery-middle cerebral artery bypass surgery was an effective and safe surgical strategy.

Keywords: Internal carotid ligation, Large-giant cavernous sinus segment aneurysms, Intermediate-flow bypass, Middle cerebral artery, superficial temporal artery.

ICA aneurysms grow slowly. In clinical practice, patients with small CS ICA aneurysms with no obvious symptoms (e.g., eye movement dysfunction or visual impairment) are usually kept under observation. ${ }^{6}$ However, a gradual increase in its size can extrude into the cranial cavity, compress the cranial nerves, which can lead to decreased 
CS ICA aneurysm
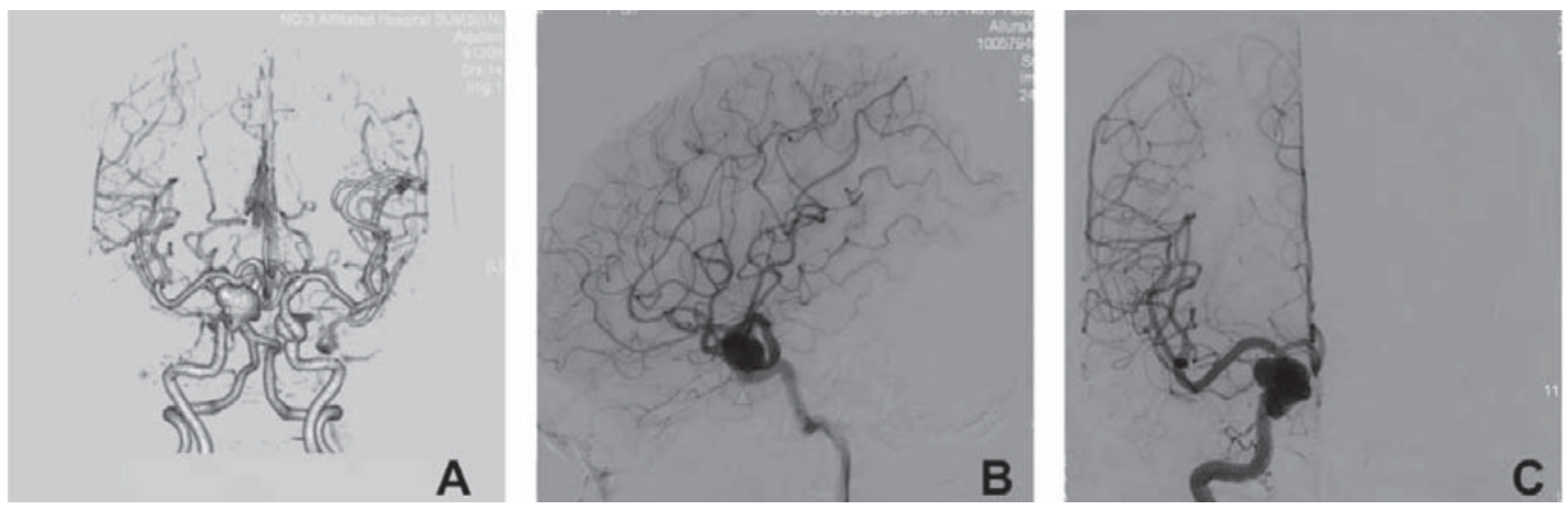

Figure 1: (A) Preoperative CTA, (B) lateral view of digital subtraction angiography, and (C) anteroposterior view of digital subtraction angiography, showing giant aneurysm on the right side of the cavernous sinus (arrowhead B-C).

visual acuity, visual field defect, or eye movement disorder. Moreover, surgical intervention is often indicated when there is a risk of subarachnoid hemorrhage. Ipsilateral ICA ligation/occlusion combined with or without EC-IC bypass has been one of the surgical strategies used for the treatment of large-giant CS ICA aneurysms. It has a low postoperative recurrence rate compared to endovascular treatment and the surgical technique requirement for EC-IC bypass is relatively simple, compared with direct clipping. There is still insufficient evidence from an established evidence-based medicine for a specific surgical strategy after ICA ligation/occlusion to address questions such as under certain scenarios. These include when to perform bypass, how to select the appropriate flow type of bypass, in which case should ICA distal to the aneurysm be clipped and many more. In this case study, we report a case of a 62-year-old woman diagnosed with large-giant CS Right ICA aneurysms, who was successfully treated with ipsilateral ICA balloon occlusion combined with high flow superficial temporal artery (STA)-Radial artery (RA)-middle cerebral artery (MCA) bypass.

\section{Case Report}

\section{Clinical manifestation}

A 62 years old woman was presented to our clinic because of dizziness for two years and oculomotor nerve palsy. Her past and family history was unremarkable. No significant positive signs were found in the neurological examination. Laboratory examinations revealed no abnormalities, and physical examination was unremarkable. Initial head Computed Tomography Angiography (CTA) demonstrated a large aneurysm on the right side of the cavernous sinus (Figure 1, A). Preoperative Digital Subtraction Angiography (DSA) confirmed the presence of a largeirregular aneurysm on the right side of the CS (Figure 1, B, C), which was $13 * 19 \mathrm{~mm}$ in size. The patient underwent carotid ultrasonography, to rule out the presence of cervical vessel stenosis. Under the supervision of a neurosurgeon, the patients were required to complete an ipsilateral Matas pressing test of the carotid artery in the clinic six times every day. Each pressing lasted for 20 minutes, and the whole training period lasted for six weeks (The disappearance of ipsilateral STA pulsation suggested that the occlusion training was valid).

\section{Balloon Occlusion Test}

Under local anesthesia, the patient underwent standard cerebral angiography and an ICA balloon occlusion test (BOT) on the affected side. After intravenous injection of 5000 UI heparin, the balloon catheter (EV3, Enfield, Connecticut, USA) was advanced to the C2 vertical body level. After the balloon inflated, contrast material was injected through the catheter into a femoral artery to confirm complete occlusion of the ICA. The BOT lasted for 30 minutes. The systolic blood pressure was reduced by $10 \%$, and the patient was observed for another 10 minutes. After balloon occlusion at Right ICA, the aneurysm disappeared, contralateral angiography showed intact anterior circulation, the middle cerebral artery trunk and branches were well developed, and the development of the lentiform artery was delayed (Figure 2, A). Contralateral ICA and/or vertebral artery angiography was also performed during the BOT process, to assess the compensation of the circle of Willis. 


\section{Bhattarai et al}
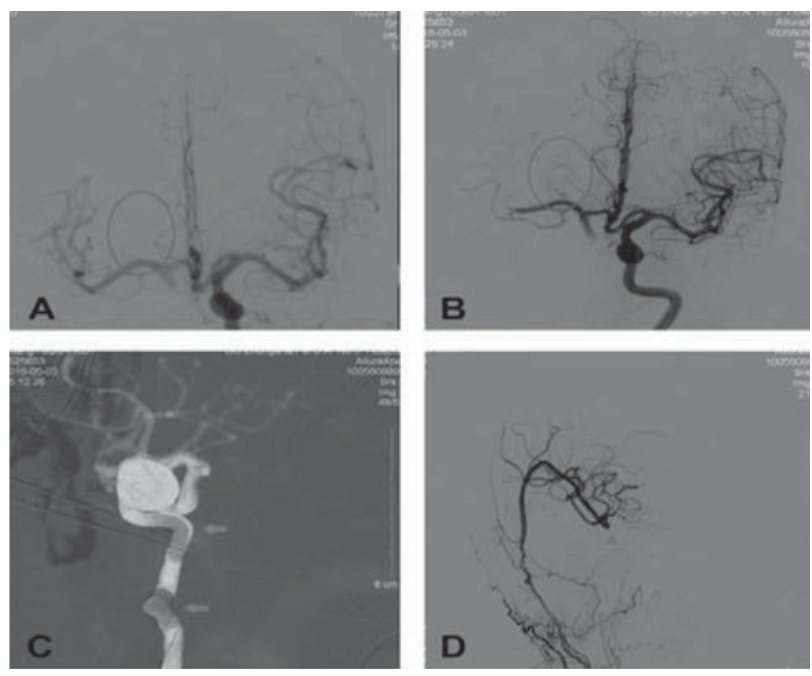

Figure 2: (A) Pre-operative contrast-enhanced angiography with ipsilateral BOT showing delayed development of lenticular artery. (B) Post-operative contrast-enhanced angiography showing the synchronization of the lenticular artery, $(C-D)$ balloon occludes the ICA without reflux (arrowhead in $C-D$ ).

\section{Treatment Strategies}

(1) DSA showed a giant aneurysm in the cavernous sinus of the Right internal carotid artery.

(2) Internal carotid artery balloon occlusion test (BOT): the blood flow of the anterior communicating artery was compensated equally. The right side imaging delay was $1.5 \mathrm{~s}-2 \mathrm{~s}$. No compensatory blood supply of posterior communicating artery was found. After blocking the right internal carotid artery for half an hour, the patient had normal left limb movement and complained of mild dizziness.

(3) Allen test: left side negative, palm collateral circulation.

(4) Strategy: right STA-RA-MCA bypass combined with internal carotid artery balloon occlusion.

\section{Surgical Procedures}

In the perioperative period (before surgery, on the day of surgery, and on the day after surgery) the patient was required to take aspirin orally $100 \mathrm{mg} /$ day. The surgery was performed under general anesthesia, and the patient received phenobarbital and hypothermia treatment $\left(35^{\circ} \mathrm{C}\right)$ to protect the brain. The intraoperative neurophysiologic monitoring system (Cadwell, Kennewick, Washington, USA) was connected. Preparation of radial artery bypass graft (RABG) should be made preoperatively. The patient was kept in a supine position, the right shoulder padded and the head rotated $60^{\circ}$ to the left. According to the
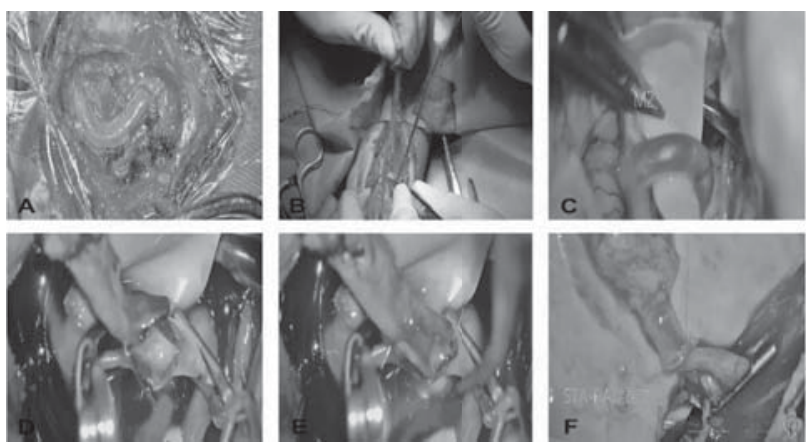

Figure 3: (A) Dissection of STA trunk, (B) Dissection of Radial artery, (C-F) intraoperative pictures of the anastomosis.

preoperative location of the superficial temporal artery, the superior auricular arc incision was made, and the main trunk of the superficial temporal artery was dissected under the microscope to be used as a feeding artery and was conserved in papaverine gauze. In the following step, the patient underwent frontopterional craniotomy. After the dura mater was opened, a long straight segment of the ipsilateral MCA M1/M2 segment intersection was exposed and isolated under microscopic observation. Then, the systolic blood pressure was increased by $20 \%$ of the baseline blood pressure. The distal end of the radial artery was anastomosed with M2 segment of MCA using 10-0 Prolene. Next, the radial artery was temporarily blocked, and the distal end of the radial artery was anastomosed with the main trunk of the STA using 8-0 Prolene(Figure 3, A-F). After the anastomosis was completed, indocyanine green angiography (ICG) was used to identify the blood flow patency. Routine angiography was performed which showed aneurysms. Two detachable balloons were used to occlude the cervical segment of the right ICA. The angiography showed that the anterior communicating artery compensated the right anterior circulation perfusion, and the right posterior communicating artery was uncompensated. The affected side was observed with electroencephalography (EEG) for half an hour. Compared with contralateral EEG activity, EEG activity of the surgical side did not show low perfusion changes, the balloon was detached. It was not necessary to expose or occlude the ICA distal to the aneurysm. The bone flap was reset, and the incisions in the head and neck were closed.

\section{Assessment of Postoperative Anastomosis and Perfusion}

The patient received head CTA + computed tomography perfusion (CTP) examinations on the first day (Figure 4, A-C) and three months (Figure 4, D-F) after the surgery, to understand the patency of vessel anastomosis and the cerebral perfusion of the surgical side. 

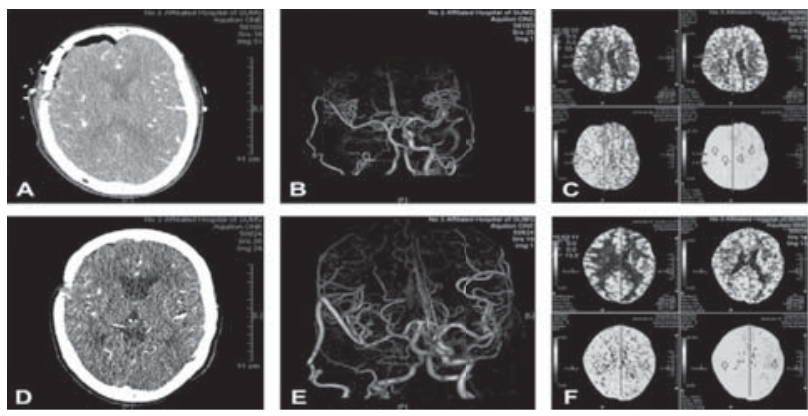

Figure 4: (A-C) Postoperative CT+CTA+CTP (1 day after surgery) respectively, the right ICA was not visualized; anastomosis was intact and communicated with the vessels. The lumen was well filled and no stenosis or occlusion was found. There was no obvious hypoperfusion in the right hemisphere, $(D-F)$ Postoperative CT $+C T A+C T P$ (90 days after surgery) respectively,results indicating thrombosis gradually formed in aneurysm lumen.

\section{Discussion}

Recent treatment options for symptomatic giant cavernous ICA aneurysms include EC-IC bypass with parent artery occlusion (PAO). , 3, , , , , 16 The favorable results of EC-IC bypass with PAO have been reported previously, $2,3,7,8,16$ although it requires the parent artery to be occluded. However, few reports have discussed the treatment and experiences of High flow bypass surgery in CS ICA aneurysm. ., 10, 17 Our case showed cranial nerve compression symptoms with higher expectations for improvement of her symptoms. Therefore, interventional embolization treatment was not considered as the preferred option. Also, although in some specific complex intracranial aneurysms flow diverters (FDs) can yield satisfactory results, our patient favored surgical treatment over FD treatment because of medical cost (FD treatment is expensive in China) and long-term efficacy (ICA ligation combined with STA-MCA has a low long-term recurrence rate). Therefore, first, ICA occlusion combined with EC-IC bypass surgery was considered as a viable surgery strategy, which supported safety at the anatomic level. Some authors have recommended surgical strategy based under single photon emission computed tomography (SPECT) results after balloon occlusion of the ipsilateral ICA. The patient should receive ICA blockage combined with EC-IC bypass surgery, if the results indicate decreased ipsilateral cerebral perfusion or otherwise, the patient should undergo simple ICA blockage. ${ }^{11,19}$ However, in our opinion, only ICA blockage may not be an ideal choice. Many studies have shown $10 \%-22 \%$ of patients showed symptoms and signs of ischemic complications (falsenegative BOT) even when the decreased cerebral perfusion
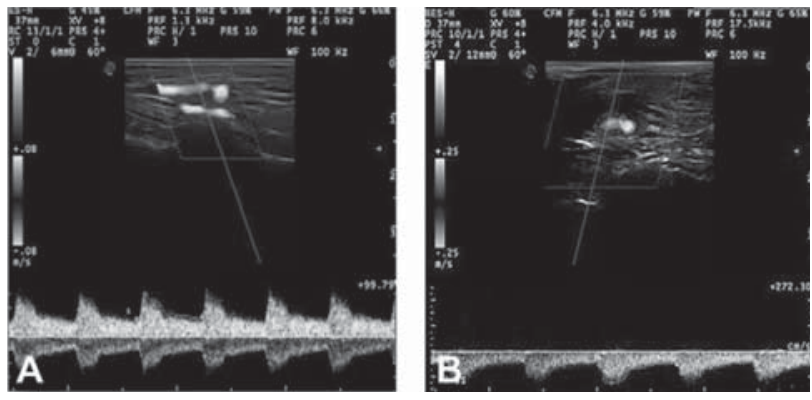

Figure 5: Postoperative TCD (A) Post-operative TCD shows blood flow of temporal branch of STA $40 \mathrm{ml} / \mathrm{min}$, (B) Post-operative TCD shows blood flow of STA trunk $90 \mathrm{ml} / \mathrm{min}$.

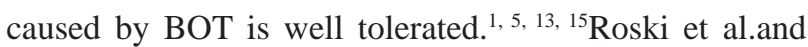
Oldershaw and Voris have also reported that the incidence of delayed ischemic complications was $1.4 \%$ and $1.9 \%$ per year, respectively. While planning EC-IC bypass with PAO for complex cerebral aneurysms. ${ }^{12,14}$ Controversy remains regarding the type of bypass, whether low flow or high flow, for each patient. The high-flow bypass is recommended to guarantee sufficient flow after PAO., 8 On the other hand; the advantages of selective cerebral revascularization with PAO have been demonstrated. ${ }^{3,16}$ Cerebral hemodynamic assessment is an important basis for selecting high flow bypass. Balloon occlusion test, in this case, showed the right middle cerebral artery and its branches developed after anterior circulation was intact, but lenticular artery development was delayed. $1 / 3^{\text {rd }}$ of the patients with good anterior communicating circulation may still have ischemic related complications after the operation. Although there was no obvious transient limb paralysis during the BOT, the delayed perfusion of the lenticular artery suggested that the compensatory blood supply of the collateral and circle of Willis might be insufficient, and occlusion of the internal carotid artery alone might lead to delayed watershed ischemia. In light of the high risk of ischemic complications in our case, the patient and we selected EC-IC bypass with PAO. Based on these findings, we used a high-flow EC-IC bypass on the dominant hemisphere, and the postoperative angiography showed the synchronization of the bilateral lenticular artery in the present case (Figure 2, B). Postoperative TCD showed that the perfusion flow of STA trunk was significantly larger than that of the STA branch. The flow of STA branch was $40 \mathrm{ml} / \mathrm{min}$, and that of STA trunk was 90ml/min (Figure 5, A-B).

Regarding ICA blockage methods, Yutaka et al. ${ }^{19}$ reported that the patients were required to receive craniotomy or endovascular treatment to achieve complete blockage of the ICA proximal and distal to the aneurysm 


\section{Bhattarai et al}

if BOT results suggested that contralateral compensatory blood refluxed back to the aneurysm cavity, This situation applied even if the ipsilateral ophthalmic artery had to be sacrificed, which could avoid regurgitated blood entering into the aneurysm lumen. Yoichi et al. ${ }^{18}$ also reported that if the BOT result suggested blood reflux into the aneurysm lumen, the aneurysm should be completely isolated with interventional embolization.

However, we disagree with this point of view. In our opinion, for all the patients, irrespective of those who showed contralateral blood reflux into the aneurysm lumen or those who did not, it was not necessary to occlude the ICA distal to the aneurysm. Following proximal ICA blockage, the blood flow and the impact to the aneurysm lumen are greatly decreased even if it is still provided with the regurgitant blood. Furthermore, in this case, the aneurysm lumen can also be regarded as a blind-ending branch after ICA blockage. Therefore, over the time thrombus formation could be observed gradually in the aneurysm cavity as a result of less and slower blood flow. The blood flow of the ipsilateral ophthalmic artery could be preserved as far as possible. The sequential results of postoperative CTA examination further corroborated our view (Figure 4, B, E).

\section{Conclusions}

We here described the case of a 62-year-old woman presented with a large aneurysm on the right side of the cavernous sinus which was successfully managed by Intermediate flow EC-IC bypass with balloon occlusion of right ICA. For patients with large-giant CS ICA aneurysms, the treatment of internal carotid occlusion combined with Intermediate-flow EC-IC bypass (STA-RA-MCA bypass) surgery was an effective and safe surgical strategy.

Abbreviations and Acronyms
BOT: Balloon occlusion test
CS: Cavernous sinus
CTA: Computed tomography angiography
CTP: Computed tomography perfusion
DSA: Digital Subtraction Angiography
EC-IC: Extracranial-Intracranial Bypass
EEG: Electroencephalography
FD: Flow diverter
ICA: Internal carotid artery
ICG: Indocyanine green
MCA: Middle cerebral artery
PAO: Parent artery occlusion
RABG: Radial artery bypass graft
SPECT: Single-photon emission computed
tomography
STA: Superficial temporal artery

\section{References}

1. Abla AA, Lawton MT. Current treatment strategies for cavernous internal carotid artery aneurysms. World Neurosurg 82:994-995, 2014

2. Date I, Ohmoto T. Long-term outcome of surgical treatment of intracavernous giant aneurysms. Neurol MedChir 38 (Suppl):62-69, 1998

3. Field M, Jungreis CA, Chengelis N, et al. Symptomatic cavernous sinus aneurysms: management and outcome after carotid occlusion and selective cerebral revascularization. AJNR Am J Neuroradiol 24:1200-1207, 2003

4. Goedee HS, Depauw PR, vdZwam B, Temmink AH. Superficial temporal artery-middle cerebral artery bypass surgery in a pediatric giant intracranial aneurysm presenting as migraine-like episodes. Childs Nerv Syst 25:257-261, 2009

5. Gonzalez CE, Moret J. Balloon occlusion of the carotid artery prior to surgery for neck tumors. AJNR Am J Neuroradiol 11:649-652, 1990

6. Kupersmith MJ, Stiebel-Kalish H, Huna-Baron R, Setton A, Niimi Y, Langer D, et al. Cavernous carotid aneurysms rarely cause subarachnoid hemorrhage or major neurologic morbidity. J Stroke Cerebrovasc Dis 11:9-14, 2002

7. Linskey ME, Sekhar LN, Hirsch WL Jr, et al. Aneurysms of the intracavernous carotid artery: natural history and indications for treatment. Neurosurgery 26:933-938, 1990

8. Linskey ME, Sekhar LN, Horton JA, et al. Aneurysms of the intracavernous carotid artery: a multidisciplinary approach to treatment. J Neurosurg 75:525-534, 1991

9. Mikabe T, Ogihara R, Tomita S, Kin H, Karasawa $\mathrm{H}$, Watanabe S, et al. Giant intracranial aneurysm visualized by prolonged injection angiography: Case report (author's transl). No Shinkei Geka 8:749753, 1980

10. Mura J, Torche E, Riquelme F, Parra M, Julio R. Three-year-old patient with giant MCA aneurysm treated by trapping-resection plus STA-MCA bypass. Case report. Childs Nerv Syst 28:169-173, 2012

11. Ohtaki S, Mikami T, Iihoshi S, Miyata K, Nonaka $\mathrm{T}$, Houkin K, et al. Strategy for the treatment of large-giant aneurysms in the cavernous portion of the internal carotid artery. No Shinkei Geka 41:107115, 2013

12. Oldershaw JB, Voris HC. Internal carotid artery ligations: a follow-up study. Neurology 16:937-938, 1966 
CS ICA aneurysm

13. Origitano TC, Al-Mefty O, Leonetti JP, DeMonte F, Reichman $\mathrm{OH}$. Vascular considerations and complications in cranial base surgery. Neurosurgery 35:351-362 [discussion: 362-363], 1994

14. Roski RA, Spetzler RF, Nulsen FE. Late complications of carotid ligations in the treatment of intracranial aneurysms. J Neurosurg 54:583-587, 1981

15. Sekhar LN, Bucur SD, Bank WO, Wright DC.Venous and arterial bypass grafts for difficult tumors, aneurysms, and occlusive vascular lesions: evolution of surgical treatment and improved graft results. Neurosurgery 44:1207-1224, 1999

16. Shimizu H, Matsumoto Y, Tominaga T. Parent artery occlusion with bypass surgery for the treatment of internal carotid artery aneurysms: clinical and hemodynamic results. Clin Neurol Neurosurg 112:32-39, 2010

17. Yang K, Ahn JS, Park JC, Kwon DH, Kwun BD, Kim CJ. The efficacy of bypass surgery using a short interposition graft for the treatment of intracranial complex aneurysm. World Neurosurg 83:197-202, 2015

18. Yoichi U, Sho O, Yoshio A, Takashi I, Noriaki M, Kinya Y. Treatment of symptomatic bilateral cavernous carotid aneurysms: long-term results of 6 cases. J Stroke Cerebrovasc Dis 24:1013-1018, 2015

19. Yutaka K, Jun-ichiro H, Motohiro M, Shigetoshi Y, Takamasa M, Jun-ichiro K, et al. Treatment strategy for giant aneurysms in the cavernous portion of the internal carotid artery. Surg Neurol 67:148-155, 2007 